\title{
Individual Differences in Blood Alcohol Concentrations after Moderate Drinking Are Mainly Regulated by Gastric Emptying Rate Together with Ethanol Distribution Volume
}

\author{
Shunji Oshima ${ }^{1}$, Takeshi Haseba ${ }^{2}$, Chiaki Masuda $^{1}$, Ema Kakimi ${ }^{1}$, Manabu Sami ${ }^{1}$, Tomomasa Kanda ${ }^{1}$, \\ Youkichi Ohno ${ }^{2}$
}

${ }^{1}$ Research Laboratories for Fundamental Technology of Food, Asahi Group Holdings, Ltd., Ibaraki, Japan; ${ }^{2}$ Department of Legal Medicine, Nippon Medical School, Tokyo, Japan.

Email: shunji.oshima@asahigroup-holdings.com

Received January $13^{\text {th }}, 2012$; revised April $5^{\text {th }}, 2012$; accepted April $13^{\text {th }}, 2012$

\begin{abstract}
Blood alcohol concentration (BAC) differs greatly among individuals, even when people of the same sex and age drink alcohol under the same drinking conditions. In this study, we investigated the main factors involved in the internal regulation of individual differences in BAC, focusing on the alcohol dehydrogenase 1B (ADH1B) genotype, blood acetaldehyde concentration $(\mathrm{BAcH})$, amount of habitual alcohol consumption, pharmacokinetic parameters of BAC, distribution volume of ethanol (Vd), and gastric emptying rate (GER) under the same drinking conditions. Twenty healthy Japanese males aged between 40 and 59 years old and having the aldehyde dehydrogenase 2 (ALDH2) genotype of ALDH 2*1/*2 were recruited for this study. The subjects were given $0.32 \mathrm{~g}$ ethanol $/ \mathrm{kg}$ body weight in the form of commercially available beer $(5 \%, \mathrm{v} / \mathrm{v})$. The results showed that BAC-max differed greatly among individuals with a more than two-fold variation. When the BAC-time curve was compared among ADH1B genotypes $\left(A D H 1 B^{*} 1 /{ }^{*} 1\right.$, $* 1 / * 2$, and $* 2 / * 2$ ), there were no differences in $\mathrm{BAC}$ among the genotypes. Although $\mathrm{BAcH}$, monthly alcohol consumption, elimination rate of blood ethanol ( $\beta$ value) and ethanol disappearance rate from the body (EDR) can affect BAC, all of them had no correlations with BAC-max. However, Vd (liter $/ \mathrm{kg}), \Delta$ Plasma glucose concentration $(\triangle \mathrm{PGC}=$ $\mathrm{PGC}_{30 \min }-\mathrm{PGC}_{0 \mathrm{~min}}$ ) and the serum concentration of gastric inhibitory polypeptide (GIP) did correlate with BAC-max. Model 2 in multiple linear regression analysis showed the optimal model for Vd and GIP with positive correlations with BAC-max. As GIP and $\triangle \mathrm{PGC}$ are both reflected by gastric emptying rate (GER), we concluded that the individual differences in BAC after moderate drinking are mainly regulated by GER together with Vd. These findings demonstrate that together with body water content, the gastrointestinal tract plays an important role in the regulation of individual differences in BAC, involving first pass metabolism of ethanol.
\end{abstract}

Keywords: Individual Differences; Blood Ethanol Concentration; Moderate Drinking; Gastric Emptying Rate; Distribution Volume

\section{Introduction}

Although light to moderate drinking is beneficial to cardiovascular health, heavy drinking often results in health and social problems [1]. Ethanol and its metabolites invoke various physiological and pharmacological effects in human bodies, which develop alcohol-related diseases. Therefore, investigation of the mechanisms of alcohol metabolism is important to regulate the levels of ethanol and its metabolites in order to diminish the harmful effects of drinking on the body. Alcohol metabolism is influenced by drinking conditions, such as the type of beverage, amount of alcohol intake, and drinking with or without food. Genetic and physiological differences in individuals also affect alcohol metabolism.

Alcohol is absorbed from the stomach and the small intestine, and is distributed throughout total body water. After alcohol distribution to the body, most of its elimination takes place by metabolic conversion via alcohol dehydrogenase $(\mathrm{ADH})$ in the liver. Acetaldehyde, the product from ethanol oxidation by $\mathrm{ADH}$, is oxidized to acetate by aldehyde dehydrogenase 2 (ALDH2). The genetic polymorphism of $A L D H 2 * 1$ and *2 is known to regulate individual tolerance to alcohol and susceptibility 
to alcohol-related diseases [2]. The $A L D H 2 * 2$ allele encoding an inactive enzyme protein is responsible for the flushing reaction caused by higher acetaldehyde concentrations after the ingestion of alcohol beverages. Individuals with this allele refrain from excessive drinking due to the adverse reactions, so it is therefore protective against alcoholism [3]. In addition, this genetic polymorphism influences the rate of alcohol metabolism due to the different blood acetaldehyde concentration $(\mathrm{BAcH})$ $[4,5]$. The genetic polymorphism of alcohol dehydrogenase 1B $\left(A D H 1 B^{*} 1, * 2\right.$ and $\left.* 3\right)$ also influences the rate of alcohol metabolism because of the differences in ADH1B activity among the genotypes [6]. The amount of habitual alcohol intake may influence the rate of alcohol metabolism because chronic alcohol consumption is known to increase the rate of alcohol metabolism [7]. Moreover, the apparent distribution volume of ethanol (Vd), which correlates with total body water content [8], can be a factor regulating blood alcohol concentration (BAC). Marshall et al. reported that Vd negatively correlated with the area under the curve of ethanol (AUC) [9]. On the other hand, it is known that first pass metabolism (FPM) in the stomach and liver influences BAC [10]. Several studies have suggested that the gastric empting rate (GER) is reduced by eating a meal, the reduction of which delays the absorption of alcohol and increases its FPM to lower BAC [11-13]. Therefore, these internal factors may regulate the levels of blood alcohol via absorption, metabolism or distribution of alcohol in the body, however, it is not clear which are main factors in internal regulation of individual BAC differences.

In this study, we investigated the main factors involved in the internal regulation of individual BAC differences, focusing on the ADH1B genotype, BAcH, amount of habitual alcohol consumption, and pharmacokinetic parameters of $\mathrm{BAC}, \mathrm{Vd}$ and GER under the same drinking conditions.

\section{Materials and Methods}

\subsection{Experimental Protocol}

The Medical Corp Jozenkai Shinagawa East One Medical Clinic Research Ethics Committee approved the proposed study, and informed consent was obtained from every participant. Twenty healthy Japanese males aged from 40 to 59 years old and with the $A L D H 2 * 1 / * 2$ genotype were recruited for this study. The $A L D H 2 * 1 / * 2$ genotype was adopted in order to investigate the influence of BAcH on BAC because people with $A L D H 2 * 1 /$ *2 exhibit various levels of $\mathrm{BAcH}$ after drinking. Amounts of monthly alcohol consumption ( $\mathrm{g} /$ month) were calculated based on the following questionnaires to each subject: average amount of daily drinking $(\mathrm{ml})$, drinking frequency, and type of alcoholic beverages consumed. The drinking frequency was divided into five categories: every day, 5 to 6 days/week, 3 to 4 days/week, 1 to 2 days/week, and less or equal 3 days/month. The subjects refrained from drinking alcohol for two days, and from eating and drinking (except water) for at least 12 hours before the test. The subjects were given $0.32 \mathrm{~g}$ ethanol $/ \mathrm{kg}$ body weight in the form of commercially available beer $(5 \%, \mathrm{v} / \mathrm{v})$.

\subsection{Blood Analyses}

Blood samples were collected from the cubital vein in each experimental period. BAC was measured using head space gas chromatography according to the methods of Okada and Mizoi [14]. BAcH was measured using a 2, 4-dinitrophenylhydrazine (DNPH) derivative method for acetaldehyde by ultraviolet detection $(365 \mathrm{~nm})$ with a HPLC technique [15]. For estimating GER, we measured both blood glucose levels and gastric inhibitory polypeptide (GIP) of a glucose-dependent insulinotropic polypeptide, because the both parameters are commonly used as indirect methods for measuring GER in various studies [16-21]. Plasma glucose concentrations (PGC, mg/dl) were determined before $(0 \mathrm{~min})$ and at $30 \mathrm{~min}$ after drinking by a hexokinase method with a routine measurement in a clinical laboratory and $\triangle \mathrm{PGC}\left(\mathrm{PGC}_{30 \text { min }}-\right.$ $\mathrm{PGC}_{0 \text { min }}$ ) was obtained ( $\triangle \mathrm{PGC}$ was used to evaluate nineteen subjects because the fasting PGC of only one subject was more than $126 \mathrm{mg} / \mathrm{dl}$ and was a diagnostic criteria of diabetes mellitus in Japan). Serum gastric inhibitory polypeptide concentrations ( $\mathrm{GIP}, \mathrm{pg} / \mathrm{ml}$ ) were measured at $30 \mathrm{~min}$ after drinking using a commercially available enzyme immunoassay kit (Human GIP Assay Kit, Immuno-Biological Laboratories Co., Ltd.).

\subsection{Ethanol Kinetic Parameters}

The elimination rate of blood ethanol ( $\beta$ value; $\mathrm{mg} / \mathrm{ml} / \mathrm{h}$ ) and ethanol disappearance rate from the body (EDR; $\mathrm{g} / \mathrm{kg} / \mathrm{h}$ ) were calculated from the slope of the pseudolinear decline of the BAC. Vd (liter $/ \mathrm{kg})$ was calculated by dividing the ethanol dose $(0.32 \mathrm{~g} / \mathrm{kg})$ by $\mathrm{C}_{0}$ ( $\mathrm{g} /$ liter $)$, which was determined from the y-intercept of the regression line.

\subsection{Regression Analyses}

We performed simple linear regression (Pearson) analyses about the correlation with BAC-max and these parameters. Then, we used a multiple linear regression analysis (stepwise method) with Dr. SPSS II software (SPSS Inc.) to examine the relationship between BACmax as a dependent variable and GIP, $\triangle \mathrm{PGC}$, and $\mathrm{Vd}$ as 
independent variables, as these three parameters indicated significant correlations with BAC-max on the simple linear regression analysis. The inclusion and exclusion criteria were set at 0.05 .

\section{Results}

\subsection{Individual Differences of BAC-Time Curves}

The BAC (means $\pm \mathrm{SD}$ ) reached $0.42 \pm 0.11 \mathrm{mg} / \mathrm{ml}$ at 30 $\min$ or $0.44 \pm 0.08 \mathrm{mg} / \mathrm{ml}$ at $60 \mathrm{~min}$ after moderate drinking of beer at an ethanol dose of $0.32 \mathrm{~g} / \mathrm{kg}$. Twenty subjects showed various levels of BAC with large individual differences; in some cases the BAC-max differed by more than twice among individuals (maximal 0.64 $\mathrm{mg} / \mathrm{ml}$ vs minimal $0.24 \mathrm{mg} / \mathrm{ml}$, Figure 1). When the BAC-time curve was compared among ADH1B genotypes $(* 1 / * 1, * 1 / * 2$, and $* 2 / * 2)$, there were no differences in BAC (Figure 2). The frequency ratio of each ADH1B genotype in this study was almost the same as that previously reported in Japanese [22]. In addition, the $\beta$ value $(\mathrm{mg} / \mathrm{ml} / \mathrm{hr})$, EDR $(\mathrm{g} / \mathrm{kg} / \mathrm{hr})$ and $\mathrm{Vd}$ (liter $/ \mathrm{kg}$ ) were not different among ADH1B genotypes $(\beta$ value; 0.13 in $* 1 / * 1,0.12 \pm 0.03$ in $* 1 / * 2,0.12 \pm 0.02$ in $* 2 / * 2$, EDR; 0.07 in $* 1 / * 1,0.08 \pm 0.01$ in $* 1 / * 2,0.07 \pm 0.01$ in $* 2 / * 2$, $\mathrm{Vd} ; 0.58$ in $* 1 / * 1,0.66 \pm 0.07$ in $* 1 / * 2,0.61 \pm 0.05$ in $* 2 / * 2$, respectively).

\subsection{Regression Analyses with BAC-max}

Simple linear regression analysis showed that BAC-max positively correlated with $\triangle \mathrm{PGC}$ and GIP, but negatively correlated with $\mathrm{Vd}$, as indicated by Pearson's correlation coefficients (Table 1). However, $\triangle \mathrm{PGC}$ was excluded by multiple linear regression analysis from model 1 and 2 (partial correlation coefficient; $0.344, p$ value; 0.176 in the model 2, Table 2). Model 2 was the optimal model in which Vd and GIP significantly correlated with BACmax. "Calculated BAC-max" values, which were calculated from the formula $\left\{0.832+0.00179 * \mathrm{GIP}-0.735^{*} \mathrm{Vd}\right\}$ obtained from model 2 correlated with the measured BAC-max with a higher multiple correlation coefficient $(0.842$, Figure 3) than that $(0.678)$ in the model 1.

\section{Discussion}

BAC greatly differed among individuals, even though the subjects were the same sex, age and drank alcohol under the same conditions (Figure 1). In this study, we investigated which factor(s) regulate individual differences in BAC. The ADH1B encoded by the $A D H 1 B^{*} 2$ allele has a greater enzymatic activity than that of the the $A D H 1 B^{*} 1$ allele [23]. However, Mizoi et al. [4] previously reported that there were no differences in $\beta_{60}$ and EDR among three ADH1B genotypes at a $0.4 \mathrm{~g} / \mathrm{kg}$ dose of ethanol,

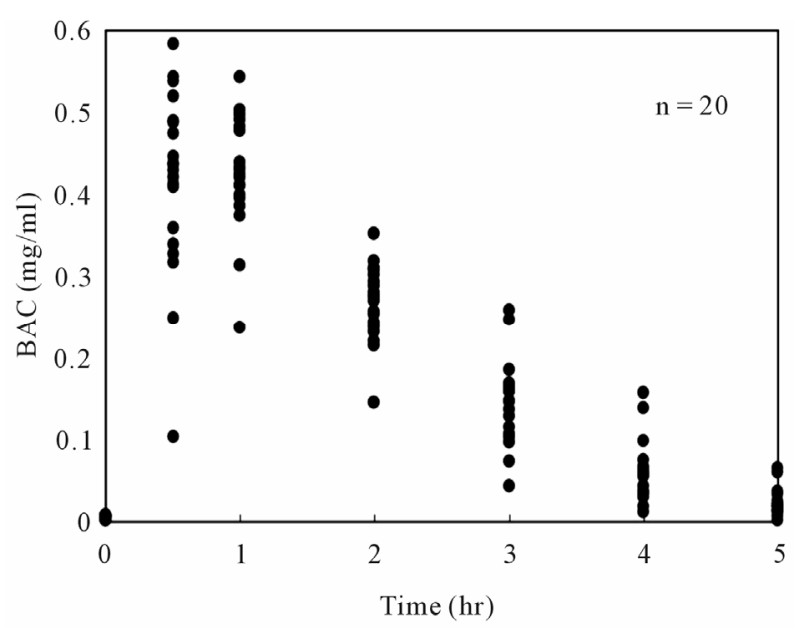

Figure 1. Blood alcohol concentrations (BAC) of each subject after moderate beer drinking.

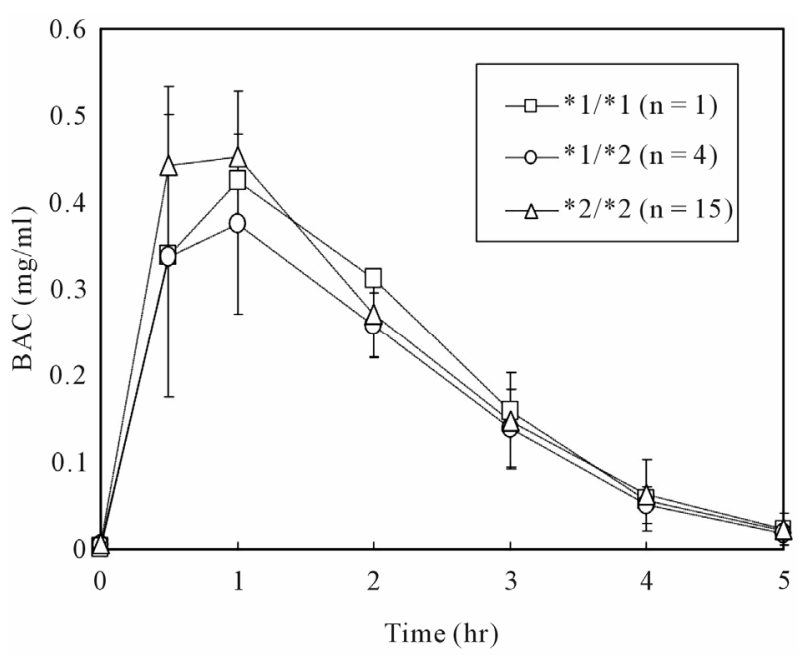

Figure 2. Blood alcohol concentrations (BAC) of each ADH1B genotype after moderate beer drinking. Values $(* 1 / * 2, * 2 / * 2)$ are means and standard deviation.

suggesting no differences in BAC-max among the genotypes. We also confirmed that there were no differences in $\beta_{60}$, EDR and BAC-max (or BAC) among the three ADH1B genotypes (Figure 2) in this study using twenty healthy males Japanese with $A L D H 2 * 1 / * 2$ genotype.

As demonstrated by the higher rate of $\beta_{60}$ and EDR in $A L D H 2 * 1 / * 1$ than $* 1 / * 2$ [4], BAcH influences alcohol metabolism by inhibiting ADH activity [24]. However, BAcH-max showed no significant correlation with BACmax for the $A L D H 2 * 1 / * 2$ genotype, as shown by the simple linear regression analysis in this study. This result suggests the differences in $\mathrm{BAcH}$ in $A L D H 2 * 1 / * 2$ genotype do not influence BAC, although the very large differences in $\mathrm{BAcH}$ seen between $A L D H 2 * 1 /{ }^{*} 1$ and $A L D H 2 * 1 / * 2$ genotypes affect BAC [5]. 
Regulated by Gastric Emptying Rate Together with Ethanol Distribution Volume

Table 1. Simple correlation coefficients of BAC-max with each parameter.

\begin{tabular}{ccccccccc}
\hline & BAcH-max & $\begin{array}{c}\text { Monthly alcohol } \\
\text { consumption }\end{array}$ & $\beta^{\mathrm{b}}$ & $\mathrm{EDR}^{\mathrm{b}}$ & $\mathrm{Vd}^{\mathrm{b}, \mathrm{c}}$ & $\Delta \mathrm{PGC}^{\mathrm{d}}$ & $\mathrm{GIP}^{\mathrm{d}}$ \\
\hline Correlation coefficient & 0.149 & 0.065 & 0.235 & -0.109 & -0.675 & 0.543 & 0.706 \\
$\quad p$ value & 0.531 & 0.785 & 0.318 & 0.647 & 0.001 & 0.016 & $<0.001$ \\
\hline
\end{tabular}

${ }^{\mathrm{a}}$ Calculated based on a questionnaire given to each subject; ${ }^{\mathrm{b}}$ Calculated from the slope of the pseudolinear decline in BAC; ${ }^{\mathrm{c}}$ Analyzed as the indicator of total body water content; ${ }^{\mathrm{d}}$ Analyzed as the indicator of GER.

Table 2. Multiple linear regression analysis (stepwise method) for BAC-max with Vd, $\triangle$ PGC and GIP.

\begin{tabular}{ccccc}
\hline Regression model & Independent variable & Partial correlation coefficient & Standardized $\beta$ & $p$ value \\
\hline $1^{\mathrm{a}}$ & $\mathrm{Vd}$ & -0.694 & -0.694 & 0.001 \\
& $\mathrm{Vd}$ & -0.711 & -0.565 & 0.001 \\
$2^{\mathrm{b}}$ & GIP & 0.661 & 0.493 & 0.003 \\
\hline
\end{tabular}

${ }^{\mathrm{a}} \Delta \mathrm{PGC}$ (partial correlation coefficient; $0.372, p$ value: 0.129 ) and GIP (partial correlation coefficient; $0.661, p$ value: 0.003 ) were excluded from the model; ${ }^{\mathrm{b}} \triangle \mathrm{PGC}$ (partial correlation coefficient; $0.344, p$ value: 0.176 ) was excluded from the model.

Amounts of monthly ethanol consumption showed no significant correlation with BAC-max either, although chronic alcohol consumption is known to increase the rate of alcohol metabolism [25].

On the other hand, $\mathrm{Vd}$ values (liter $/ \mathrm{kg}$ ) was shown to relate to the individual BAC differences with negative correlations by both simple and multiple linear regression analyses (Tables 1 and 2). Although Vd generally indicate the total body water content (liter $/ \mathrm{kg}$ ) of each subject [8], it is changed by drinking conditions via the FPM of ethanol, because it is calculated using the theoretical maximal concentration of blood ethanol $\left(\mathrm{C}_{0}\right)$. When FPM occurs after drinking, the actual amounts of ethanol into the systemic circulation are lower than the dosage. For example, $\mathrm{Vd}$ in the case of drinking with a meal increases more than drinking without a meal because the meal increases FPM. Therefore, total body water content directly measured may be more accurate than $\mathrm{Vd}$ to evaluate the factors regulating the differences in individual BAC. FPM in the digestive tract or liver is another factor that affects BAC [26-28]. BAC has been shown to be lower [29] when FPM occurs in the stomach, where two types of ADH isozymes (ADH 3 and 4) are located and contribute to FPM [30]. GER is an important factor in the FPM [31]. Johnson et al. [32] reported that cigarette smoking slowed GER and was associated with reductions in BAC-max. Oneta et al. [33] also reported in a human study that the administration of N-butylscopolamine increased the gastric FPM of ethanol by delaying GER, probably due to an increase in time of ethanol exposure to gastric $\mathrm{ADH}$ isozymes. $\triangle \mathrm{PGC}$ has a positive correlation with GER without directly affecting the gastrointestinal tract [19]. GIP was also shown to be associated with GER because GIP is excreted after nutrients

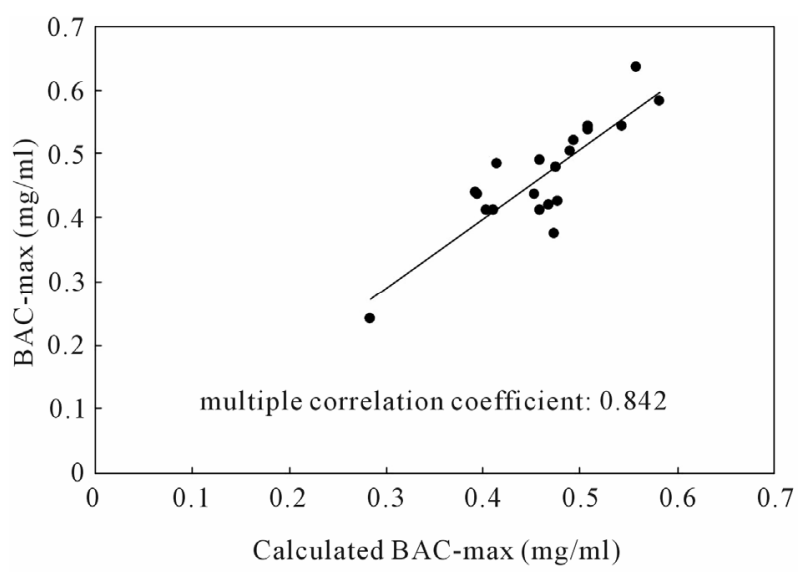

Figure 3. Correlation of BAC-max and calculated BACmax by multiple linear regression analysis. Closed circles indicate each subject's data. Calculated BAC-max values were calculated following a formula which was obtained by multiple regression analysis: $0.832+0.00179 * \mathrm{GIP}(\mathrm{pg} / \mathrm{ml})-$ $0.735 * V d$ (liter/kg).

reach the intestinal tract from the gut $[16,17]$. The both parameters have been frequently used in various studies dealing with gastric emptying [16-21]. Therefore, serum GIP and $\triangle \mathrm{PGC}$ were adopted as the parameters of GER in this study. The two parameters significantly correlated with BAC-max (Table 1), indicating that GER regulates individual differences in BAC-max. Moreover, we calculated the "Calculated BAC-max" from the formula $\left\{0.832+0.00179 * \mathrm{GIP}-0.735^{*} \mathrm{Vd}\right\}$, which was obtained by multiple regression analysis, because GIP and $\mathrm{Vd}$ were selected, but $\triangle \mathrm{PGC}$ was excluded, by the analysis in regression model 2. GIP and $\triangle \mathrm{PGC}$ are factors which increase after food materials reach duodenum from stomach, but each maximal increases in the blood 
have temporal difference $[34,35]$, therefore, we speculated that the correlation of both parameters with BACmax was different. As shown to correlation to BAC-max, GIP may be a better indicator than $\triangle \mathrm{PGC}$. As a result, the "calculated BAC-max" strongly correlated with the measured BAC-max with a higher multiple correlation coefficient (0.842, in Figure 3).

\section{Conclusion}

We concluded that the individual differences in BAC after moderate drinking are mainly regulated by GER together with Vd. Thus, the gastrointestinal tract plays an important role in the regulation of individual differences in BAC via FPM, together with the total body water content.

\section{REFERENCES}

[1] R. Guo and J. Ren, "Alcohol and Acetaldehyde in Public Health: From Marvel to Menace," International Journal of Environmental Research and Public Health, Vol. 7, No. 4, 2010, pp. 1285-1301.

http://www.mdpi.com/1660-4601/7/4/1285/

doi:10.3390/ijerph7041285

[2] S. Harada, D. P. Agarwal and H. W. Goedde, "Aldehyde Dehydrogenase Deficiency as Cause of Facial Flushing Reaction to Alcohol in Japanese," Lancet, Vol. 2, No. 8253, 1981, p. 982. doi:10.1016/S0140-6736(81)91172-7

[3] G. S. Peng, M. F. Wang, C. Y. Chen, S. U. Luu, H. C. Chou, T. K. Li and S. J. Yin, "Involvement of Acetaldehyde for Full Protection against Alcoholism by Homozygosity of the Variant Allele of Mitochondrial Aldehyde Dehydrogenase Gene in Asians," Pharmacogenetics, Vol. 9, No. 4, 1999, pp. 463-476.

[4] Y. Mizoi, K. Yamamoto, Y. Ueno, T. Fukunaga and S. Harada, "Involvement of Genetic Polymorphism of Alcohol and Aldehyde Dehydrogenases in Individual Variation of Alcohol Metabolism," Alcohol and Alcoholism, Vol. 29, No. 6, 1994, pp. 707-710. http://alcalc.oxfordjournals.org/content/29/6/707.long

[5] S. Oshima, T. Haseba, C. Masuda, Y. Abe, M. Sami, T. Kanda and Y. Ohno, "Alcohol Metabolism at Moderate Drinking in Healthy Men. Comparison between Differences of Alcohol Beverages, with and without Meal, and Genetic Polymorphism," Japanese Journal of Alcohol Studies and Drug Dependence, Vol. 46, No. 3, 2011, pp. 357-367.

[6] H. R. Thomasson, J. D. Beard and T. K. Li, “ADH2 Gene Polymorphisms Are Determinants of Alcohol Pharmacokinetics," Alcoholism: Clinical and Experimental Research, Vol. 19, No. 6, 1995, pp. 1494-1499. doi:10.1111/j.1530-0277.1995.tb01013.x

[7] C. S. Lieber, L. M. DeCarli, L. Feinman, Y. Hasumura, M. Korsten, S. Matsuzaki and R. Teschke, "Effect of Chronic Alcohol Consumption on Ethanol and Acetaldehyde Metabolism," Advances in Experimental Medicine and Biology, Vol. 59, 1975, pp. 185-227.

[8] A. H. Tzamaloukas, J. E. Jackson, J. C. Gallegos, D. A. Long and M. M. McLane, "Distribution Volume of Ethanol as a Measure of Body Water," Mineral and Electrolyte Metabolism, Vol. 11, No. 2, 1985, pp. 123-130.

[9] A. W. Marshall, D. Kingstone, M. Boss and M. Y. Morgan, "Ethanol Elimination in Males and Females: Relationship to Menstrual Cycle and Body Composition," Hepatology, Vol. 3, No. 5, 1983, pp. 701-706. doi:10.1002/hep. 1840030513

[10] R. T. Gentry, E. Baraona and C. S. Lieber, "Agonist: Gastric First Pass Metabolism of Alcohol," Journal of Laboratory and Clinical Medicine, Vol. 123, No. 1, 1994, pp. 21-26.

[11] R. P. Roine, R. T. Gentry, R. T. Lim Jr., E. Helkkonen, M. Salaspuro and C. S. Lieber, "Comparison of Blood Alcohol Concentrations after Beer and Whiskey," Alcoholism: Clinical and Experimental Research, Vol. 17, No. 3, 1993, pp. 709-711. doi:10.1111/j.1530-0277.1993.tb00824.x

[12] R. T. Lim Jr., R. T. Gentry, D. Ito, H. Yokoyama, E. Baraona and C. S. Lieber, "First-Pass Metabolism of Ethanol Is Predominantly Gastric," Alcoholism: Clinical and Experimental Research, Vol. 17, No. 6, 1993, pp. 13371344. doi:10.1111/j.1530-0277.1993.tb05250.x

[13] S. Oshima, C. Masuda, E. Kakimi, M. Sami, T. Kanda, T. Haseba and Y. Ohno, "Gastric First Pass Metabolism Plays an Important Role in the Food-Induced Lowering of Blood Alcohol Concentration and AUC, Enhanced by the Delay of Gastric Empting," Alcohol and Biomedical Research, Vol. 30, 2011, pp. 64-71.

[14] T. Okada and Y. Mizoi, "Studies on the Problem of Blood Acetaldehyde Determination in Man and Level after Alcohol Intake," Japanese Journal of Alcohol Studies and Drug Dependence, Vol. 17, No. 2, 1982, pp. 141-159.

[15] D. Kozutsumi, M. Arita, A, Kawashima, M, Adachi and M. Takami, "An Improved Method for Acetaldehyde Determination in Blood by High-Performance Liquid Chromatography and Solid-Phase Extraction," Journal of Chromatographic Science, Vol. 40, No. 10, 2002, pp. 477482.

[16] J. M. Polak, S. R. Bloom, M. Kuzio, J. C. Brown and A. G. Pearse, "Cellular Localization of Gastric Inhibitory Polypeptide in the Duodenum and Jejunum," Gut, Vol. 14, No. 4, 1973, pp. 284-288. doi:10.1136/gut.14.4.284

[17] I. G. Cleator and R. H. Gourlay, "Release of Immunoreactive Gastric Inhibitory Polypeptide (IR-GIP) by Oral Ingestion of Food Substances," American Journal of Surgery, Vol. 130, No. 2, 1975, pp. 128-135. doi:10.1016/0002-9610(75)90360-8

[18] K. B. Lauritsen, H. J. Frederiksen, A. Uhrenholdt and J. J. Holst, "The Correlation between Gastric Emptying Time and the Response of GIP and Enteroglucagon to Oral Glucose in Duodenal Ulcer Patients," Scandinavian Journal of Gastroenterology, Vol. 17, No. 4, 1982, pp. 513516. doi:10.3109/00365528209182241

[19] M. Horowitz, M. A. Edelbroek, J. M. Wishart and J. W. Straathof, "Relationship between Oral Glucose Tolerance 
and Gastric Emptying in Normal Healthy Subjects," Diabetologia, Vol. 36, No. 9, 1993, pp. 857-862. doi:10.1007/BF00400362

[20] A. Karamanlis, R. Chaikomin, S. Doran, M. Bellon, F. D. Bartholomeusz, J. M. Wishart, K. L. Jones, M. Horowitz and C. K. Rayner, "Effects of Protein on Glycemic and Incretin Responses and Gastric Emptying after Oral Glucose in Healthy Subjects," American Journal of Clinical Nutrition, Vol. 86, No. 5, 2007, pp. 1364-1368. http://www.ajcn.org/content/86/5/1364.long

[21] J. E. Stevens, O. H. Gilja, D. Gentilcore, T. Hausken, M. Horowitz and K. L. Jones, "Measurement of Gastric Emptying of a High-Nutrient Liquid by 3D Ultrasonography in Diabetic Gastroparesis," Neurogastroenterology and Motility, Vol. 23, No. 3, 2011, pp. 220-225. doi:10.1111/j.1365-2982.2010.01630.x

[22] M. Y. Eng, S. E. Luczak and T. L. Wall, "ALDH2, ADH1B, and ADH1C Genotypes in Asians: A Literature Review," Alcohol Research \& Health, Vol. 30, No. 1, 2007, pp. 22-27.

[23] H. J. Edenberg, "Regulation of the Mammalian Alcohol Dehydrogenase Genes," Progress in Nucleic Acid Research \& Molecular Biology, Vol. 64, 2000, pp. 295-341. doi:10.1016/S0079-6603(00)64008-4

[24] A. G. Dawson, "Ethanol Oxidation in Systems Containing Soluble and Mitochondrial Fractions of Rat Liver. Regulation by Acetaldehyde," Biochemical Pharmacology, Vol. 32, No. 14, 1983, pp. 2157-2165. doi:10.1016/0006-2952(83)90221-6

[25] Y. Ueno, J. Adachi, H. Imamichi, A. Nishimura and Y. Tatsuno, "Effect of the Cytochrome P-450IIE1 Genotype on Ethanol Elimination Rate in Alcoholics and Control Subjects," Alcoholism: Clinical and Experimental Research, Vol. 20, No. 1, 1996, pp. 17A-21A. doi:10.1111/j.1530-0277.1996.tb01720.x

[26] S. Kechagias, K. A. Jönsson and A. W. Jones, "Impact of Gastric Emptying on the Pharmacokinetics of Ethanol as Influenced by Cisapride," British Journal of Clinical Pharmacology, Vol. 48, No. 5, 1999, pp. 728-732. doi:10.1046/j.1365-2125.1999.00080.x

[27] A. Franke, I. A. Nakchbandi, A. Schneider, H. Harder and M. V. Singer, "The Effect of Ethanol and Alcoholic Beverages on Gastric Emptying of Solid Meals in Humans,"
Alcohol and Alcoholism, Vol. 40, No. 3, 2005, pp. $187-$ 193. doi:10.1093/alcalc/agh138

[28] K. L. Wu, R. Chaikomin, S. Doran, K. L. Jones, M. Horowitz and C. K. Rayner, "Artificially Sweetened versus Regular Mixers Increase Gastric Emptying and Alcohol Absorption," American Journal of Medicine, Vol. 119, No. 9, 2006, pp. 802-804. doi:10.1016/j.amjmed.2006.02.005

[29] J. Caballeria, M. Frezza, R. Hernández-Muñoz, C. DiPadova, M. A. Korsten, E. Baraona and C. S. Lieber, "Gastric Origin of the First-Pass Metabolism of Ethanol in Humans: Effect of Gastrectomy," Gastroenterology, Vol. 97, No. 5, 1989, pp. 1205-1209.

[30] D. M. Pestalozzi, R. Bühler, J. P. von Wartburg and M. Hess, "Immunohistochemical Localization of Alcohol Dehydrogenase in the Human Gastrointestinal Tract," Gastroenterology, Vol. 85, No. 5, 1983, pp. 1011-1016.

[31] H. K. Seitz and G. Pöschl, "The Role of Gastrointestinal Factors in Alcohol Metabolism," Alcohol and Alcoholism, Vol. 32, No. 5, 1997, pp. 543-549. doi:10.1093/oxfordjournals.alcalc.a008294

[32] R. D. Johnson, M. Horowitz, A. F. Maddox, J. M. Wishart and D. J. Shearman, "Cigarette Smoking and Rate of Gastric Emptying: Effect on Alcohol Absorption," British Medical Journal, Vol. 302, No. 20, 1991, pp. 20-23. doi:10.1136/bmj.302.6767.20

[33] C. M. Oneta, U. A. Simanowski, M. Martinez, A. Allali-Hassani, X. Parés, N. Homann, C. Conradt, R. Waldherr, W. Fiehn, C. Coutelle and H. K. Seitz, "First Pass Metabolism of Ethanol Is Strikingly Influenced by the Speed of Gastric Emptying," Gut, Vol. 43, No. 5, 1998, pp. 612-619. doi:10.1136/gut.43.5.612

[34] K. B. Lauritsen and A. J. Moody, "The Response of Gastric Inhibitory Polypeptide (GIP) and Insulin to Glucose in Duodenal Ulcer Patients," Diabetologia, Vol. 14, No. 3, 1978, pp. 149-153. doi:10.1007/BF00429773

[35] D. Gentilcore, R. Chaikomin, K. L. Jones, A. Russo, C. Feinle-Bisset, J. M. Wishart, C. K. Rayner and M. Horowitz, "Effects of Fat on Gastric Emptying of and the Glycemic, Insulin, and Incretin Responses to a Carbohydrate Meal in Type 2 Diabetes," Journal of Clinical Endocrinology \& Metabolism, Vol. 91, No. 6, 2006, pp. 2062 2067. doi:10.1210/jc.2005-2644 